\title{
Iris Recognition under Alcohol Influence: A Preliminary Study
}

\author{
Sunpreet S. Arora, Mayank Vatsa, Richa Singh \\ IIIT Delhi \\ New Delhi, India \\ \{sunpreet08053, mayank, rsingh\}@iitd.ac.in
}

\author{
Anil Jain \\ Michigan State University \\ East Lansing, USA \\ jain@cse.msu.edu
}

\begin{abstract}
Iris recognition has been used mainly to recognize cooperative subjects in controlled environments. With the continuing improvements in iris matching performance and reduction in the cost of iris scanners, the technology will witness broader applications and may be confronted with newer challenges. In this research, we have investigated one such challenge, namely matching iris images captured before and after alcohol consumption. Due to alcohol consumption, the pupil dilates/constricts which causes deformation in iris pattern, possibly affecting iris recognition performance. The experiments performed on the "IIITD Iris Under Alcohol Influence" database show that in matching pre and post alcohol consumption images, the overlap between genuine and impostor match score distributions increases by approximately $20 \%$. These results on a relatively small database suggest that about one in five subjects under alcohol influence may be able to evade identification by iris recognition.
\end{abstract}

\section{Introduction}

Among various biometric modalities, iris, arguably, is one of the most reliable, universal, measurable, accurate and inimitable. In 1985, iris recognition was first proposed by Flom and Safir [13]. In 1993, Daugman proposed an iris recognition algorithm representing iris image as a mathematical function [11]. After that Wildes [21], Boles [8], Ma [16], and several other researchers proposed different recognition algorithms [9], [12], [17], [20]. Over the years, it has been established that every iris is unique, particularly in the detailed structure of the front or anterior layer. Not only are the irides of identical twins different, but the iris of the two eyes of the same person are also different. Although specific details of the appearance of an iris vary depending on the level and direction of illumination, it has been claimed that the basic and significant features of iris remain stable and do not change over a long time. These properties have led to the use of iris in several large-scale civilian ap-
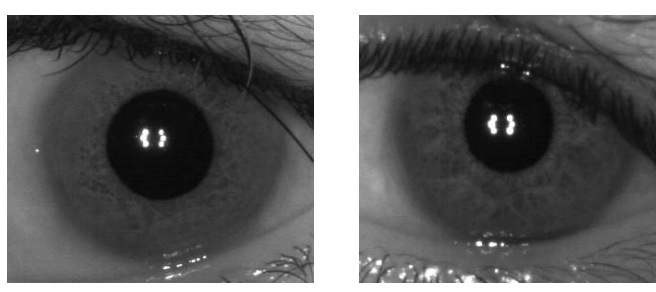

Pre Alcohol Consumption

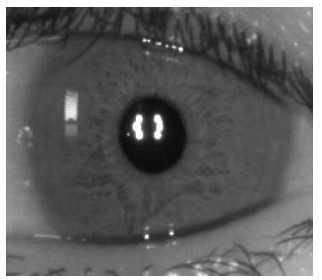

Pupil Constriction

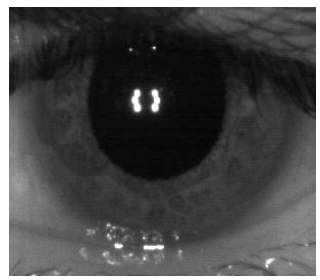

Pupil Dilation
Post Alcohol Consumption
Figure 1. Examples illustrating constriction and dilation in pupils due to alcohol consumption.

plications such as the NEXUS pass program [4], UK IRIS program [6], and India's UID program [5] for authentication/identification purposes.

Generally, covariates in iris recognition are image quality (i.e. noise, blur), illumination (specular reflection), offangle, occlusion, and resolution [15]. In recent years, several approaches have been developed to advance the stateof-art in iris recognition and address these covariates [19]. However, there can be potentially many other covariates in iris recognition which have not yet been identified. In our opinion, iris recognition under the influence of alcohol is one such covariate. Usually, it is presumed that pupil dilates after alcohol consumption [1] whereas some other research suggests that alcohol consumption does not affect iris recognition [2], [3]. Similarly, Brown et al. [10] suggest that alcohol produces no effect on pupil size whereas Richman et al. [18] suggest that drug recognition experts use pupil size for detecting subjects under alcohol/drug influence. On the other hand, medical literature suggests that, the pupil dilates up to a certain limit of alcohol consumption and thereafter it starts constricting [14]. The limits of dila- 
tion and constriction are not the same for all subjects; rather they depend on the individual's anatomy and other medical conditions. Figure 1 shows some examples of pupil dilation and constriction after alcohol consumption. Let us assume that one image (pre-alcohol) is used as the gallery and the other image (post-alcohol) is used as the probe. Due to large dilation/constriction, there is a change in iris information content and therefore, iris recognition algorithms may not be able to correctly match them. To the best of our knowledge, there has not been any study that has carefully evaluated iris recognition performance under alcohol influence. We conjecture that this problem has not received adequate attention for the following reasons:

- Minor dilation and constriction ${ }^{1}$ of the iris can be addressed with polar coordinate transformation.

- It is assumed that the subjects are cooperative and not under the alcohol influence.

- Lack of a database and challenges in collecting such as database to analyze the effect of alcohol on iris recognition.

In this paper, we introduce alcohol influence as one of the covariates in iris recognition. Major contributions of this work are: (a) collect the Iris Under Alcohol Influence (IUAI) database with 110 unique irises, (b) illustrate that large pupil dilation and constriction due to alcohol influence affects iris recognition, and (c) a simple approach to measure dilation and constriction in pupil and iris boundaries.

\section{Database}

Collecting a database that contains iris images captured before and after alcohol consumption is a challenging task. To the best of our knowledge, there is no public database that contains such variations for multiple subjects. In this research, the first contribution is collecting the IIITD Iris under Alcohol Influence (IUAI) database ${ }^{2}$.

Iris images were captured using the Vista iris scanner ${ }^{3}$ in near infrared domain under semi-controlled environment. A total of 55 subjects volunteered (age group of 25-75 years) and two images of both the eyes were captured before and after alcohol consumption. The subjects consumed about $200 \mathrm{ml}$ of alcohol (with $42 \%$ concentration level) in approximately 15 minutes and the images were captured 15-20 minutes after alcohol consumption. In this process, room temperature, lighting and other factors were kept constant

\footnotetext{
${ }^{1}$ Constriction and contraction are used interchangeably in the paper.

${ }^{2}$ The database will be made available at http://research.iiitd.edu.in/groups/iab/resources.html.

${ }^{3}$ http://www.neurotechnology.com/eye-iris-scanner-vistafa2e.html
}

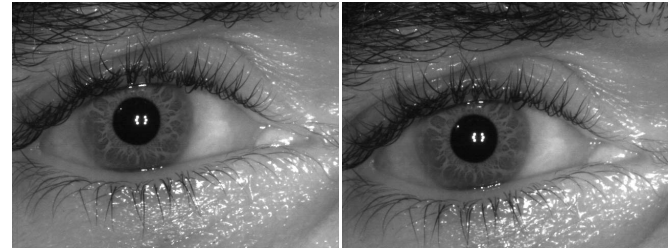

(a)

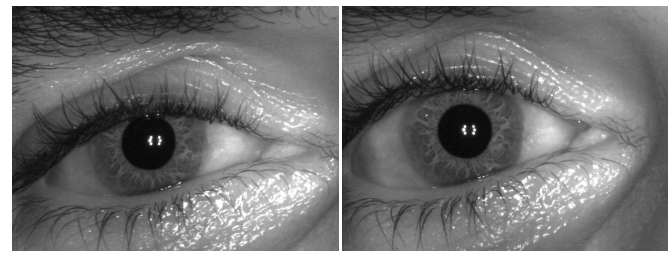

(b)

Figure 2. Sample images from the Iris Under Alcohol Influence database (a) pre-alcohol consumption images and (b) post-alcohol consumption images of two different irides.

so that the only varying parameter was alcohol consumption. The database contains 220 pre alcohol and 220 post alcohol images obtained from 55 subjects. Assuming the iris patterns of two eyes of a person to be independent, there are 110 iris classes with each class having two pre and two post alcohol images. Figure 2 shows an example of the IIITD IUAI database.

\section{Measuring Pupil Dilation or Contraction}

For measuring the extent of pupil dilation or contraction (after alcohol consumption), we have designed a simple yet effective algorithm. Let $I_{P r e}$ be the iris image of a subject $S$ taken before alcohol consumption and $I_{\text {Post }}$ be the iris image of the same subject captured after alcohol consumption. The iris and pupil boundaries obtained after iris segmentation are assumed to be of elliptical shape, and the major and minor axis of each of the ellipses is computed.

For pre-consumption image $\left(I_{\text {Pre }}\right)$, let $L P_{\text {major }}\left(I_{\text {Pre }}\right)$ and $L P_{\text {minor }}\left(I_{\text {Pre }}\right)$ be the length of the major and minor axis of pupil boundaries, respectively. Similarly, let $L I_{\text {major }}\left(I_{\text {Pre }}\right)$ and $L I_{\text {minor }}\left(I_{\text {Pre }}\right)$ be the length of the major and minor axis of iris boundaries, respectively. $L P_{\text {major }}\left(I_{\text {Post }}\right), L P_{\text {minor }}\left(I_{\text {Post }}\right), L I_{\text {major }}\left(I_{\text {Post }}\right)$, and $L I_{\text {minor }}\left(I_{\text {Post }}\right)$ represent the length of major and minor axis of pupil and iris boundaries of post alcohol consumption image $\left(I_{\text {Post }}\right)$, respectively. The area of inner ellipse or pupil boundary for both $I_{P r e}$ and $I_{\text {Post }}$ can be computed by:

$$
\begin{gathered}
A_{\text {Pupil }}\left(I_{\text {Pre }}\right)=\pi * \frac{L P_{\text {major }}\left(I_{\text {Pre }}\right)}{2} * \frac{L P_{\text {minor }}\left(I_{\text {Pre }}\right)}{2} \\
A_{\text {Pupil }}\left(I_{\text {Post }}\right)=\pi * \frac{L P_{\text {major }}\left(I_{\text {Post }}\right)}{2} * \frac{L P_{\text {minor }}\left(I_{\text {Post }}\right)}{2}
\end{gathered}
$$


Similarly, the area of the outer ellipse or the iris boundary can be computed using the following equation.

$$
\begin{gathered}
A_{\text {Iris }}\left(I_{\text {Pre }}\right)=\pi * \frac{L I_{\text {major }}\left(I_{\text {Pre }}\right)}{2} * \frac{L I_{\text {minor }}\left(I_{\text {Pre }}\right)}{2} \\
A_{\text {Iris }}\left(I_{\text {Post }}\right)=\pi * \frac{L I_{\text {major }}\left(I_{\text {Post }}\right)}{2} * \frac{L I_{\text {minor }}\left(I_{\text {Post }}\right)}{2}
\end{gathered}
$$

Let $M_{\text {Iris }}\left(I_{\text {Pre }}\right)$ denote the mask area for the preconsumption image, i.e. the fraction of the iris area which is occluded by eyelids. Similarly, let $M_{\text {Iris }}\left(I_{\text {Post }}\right)$ be the mask area for the post alcohol consumption image. The iris area which is not occluded in the pre and post alcohol consumption images, $U A_{\text {Iris }}\left(I_{\text {Pre }}\right)$ and $U A_{\text {Iris }}\left(I_{\text {Post }}\right)$, respectively, can be computed as:

$$
\begin{gathered}
U A_{\text {Iris }}\left(I_{\text {Pre }}\right)=\left(1-M_{\text {Iris }}\left(I_{\text {Pre }}\right)\right) * A_{\text {Iris }}\left(I_{\text {Pre }}\right) \\
U A_{\text {Iris }}\left(I_{\text {Post }}\right)=\left(1-M_{\text {Iris }}\left(I_{\text {Post }}\right)\right) * A_{\text {Iris }}\left(I_{\text {Post }}\right)
\end{gathered}
$$

The usable area for iris recognition is then computed as follows.

$$
\begin{array}{r}
R\left(I_{\text {Pre }}\right)=U A_{\text {Iris }}\left(I_{\text {Pre }}\right)-A_{\text {Pupil }}\left(I_{\text {Pre }}\right) \\
R\left(I_{\text {Post }}\right)=U A_{\text {Iris }}\left(I_{\text {Post }}\right)-A_{\text {Pupil }}\left(I_{\text {Post }}\right)
\end{array}
$$

Since this area is the difference of areas of the two ellipses on the absolute scale, we normalize it using Equation 5.

$$
\begin{aligned}
N R\left(I_{\text {Pre }}\right) & =\frac{R\left(I_{\text {Pre }}\right)}{A_{\text {Iris }}\left(I_{\text {Pre }}\right)} \\
N R\left(I_{\text {Post }}\right) & =\frac{R\left(I_{\text {Post }}\right)}{A_{\text {Iris }}\left(I_{\text {Post }}\right)}
\end{aligned}
$$

The final step involves taking the ratio of these normalized areas and obtaining the extent of change for a subject, $S$, due to alcohol consumption.

$$
E(S)=\frac{N R\left(I_{\text {Pre }}\right)}{N R\left(I_{\text {Post }}\right)}
$$

The extent of change, as the name suggests, is a relative measure of change in the usable area for iris recognition before and after the consumption of alcohol. Clearly, if $N R\left(I_{\text {Pre }}\right)$ is greater than $N R\left(I_{\text {Post }}\right)$, the extent of change would be greater than one, and hence signify pupil dilation after alcohol consumption. Similarly, if $N R\left(I_{\text {Pre }}\right)$ is less than $N R\left(I_{\text {Post }}\right)$, the extent of change would be less than one, signifying pupil contraction. This can also be written as,

$$
\text { Result }= \begin{cases}\text { Dilation } & \text { if } E(S)>1 \\ \text { Constriction } & \text { if } E(S)<1 \\ \text { No change } & \text { Otherwise. }\end{cases}
$$

Note that the proposed algorithm can also be used to compute the extent of change due to dilation/contraction caused by other factors such as illumination. In this research, it is used to measure the effect of alcohol consumption.

\section{Results and Analysis}

The effect of alcohol consumption on the IIITD IUAI database is studied using the approach discussed in Section 3. This section discusses the algorithms used to analyze the matching performance, experimental protocol, and analysis.

\subsection{Algorithms and Experimental Protocol}

For experiments and analysis, two iris recognition algorithms are used: (1) iris segmentation, feature extraction and matching algorithm by Vatsa et al. [20] ${ }^{4}$ and (2) VeriEye commercial system [7].

Three types of experiments are performed to understand the effect of alcohol consumption on the performance of iris recognition algorithms.

1. Matching two pre alcohol consumption iris images.

2. Matching two post alcohol consumption iris images.

3. Matching pre and post alcohol consumption iris images.

The first two experiments are conducted to establish the baseline performance on this database and the third experiment is conducted to analyze the effect of alcohol consumption.

\subsection{Analysis}

Figures 3 and 4 show the match score distributions obtained from Vatsa et al.'s algorithm [20] and VeriEye, respectively. Key observations are summarized below.

- Using the extent of change algorithm described in Section 3, it is observed that among pre-post iris pair comparisons, the consumption of alcohol led to dilation in about $51 \%$ of the cases, whereas contraction is observed in the remaining $49 \%$ of the cases. This suggests that there is almost an equal chance of iris getting dilated or contracted under alcohol influence. Further, there are about $32 \%$ cases of contraction and $24 \%$ cases of dilation beyond the tolerance level ${ }^{5}$.

\footnotetext{
${ }^{4}$ It is to be noted that we have not used matchers that are available online because the code yields several segmentation errors for this database.

${ }^{5}$ Tolerance is defined as an indicator of $\mathrm{E}(\mathrm{S})$ values for which the match scores lie in the tail of the distribution - beyond mean \pm one standard deviation.
} 


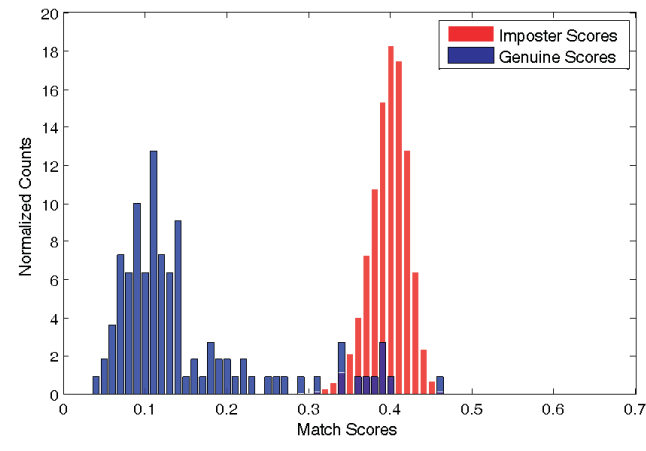

(a)

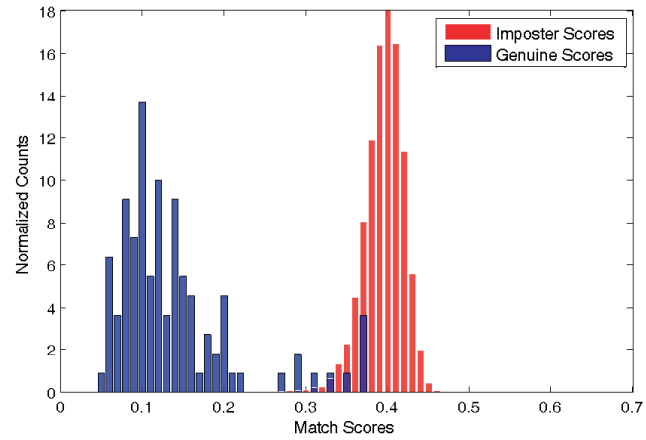

(b)

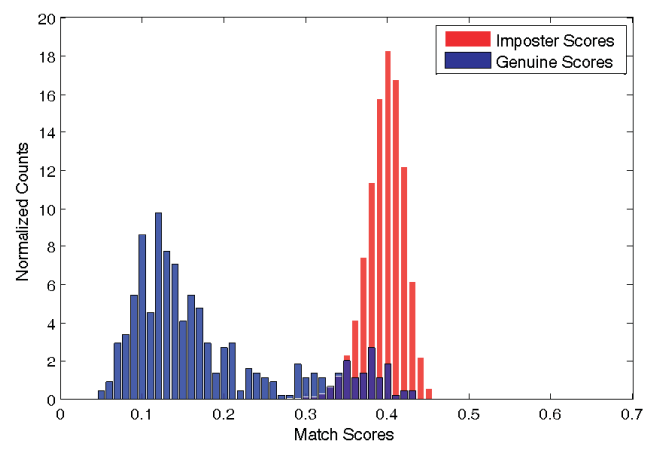

(c)

Figure 3. Genuine and impostor match score distribution obtained by using $2 \mathrm{D} \log$ polar Gabor wavelet based feature encoding and SVM based matching [20] (a) both gallery and probe are captured before consumption, (b) both gallery and probe are captured after consumption, and (c) gallery is captured before consumption and probe is the image captured after consumption.

- The average pupil diameter changes by about nine pixel units (on a scale of 90-100 pixel units). In other words, it decreases or increases in this range for both horizontal and vertical ellipsoidal diameters. On the other hand, this variation is only about two pixels for the iris diameter (on a scale of around 250 pixel units). This establishes that the inner boundary of iris changes significantly more than the outer boundary even on a

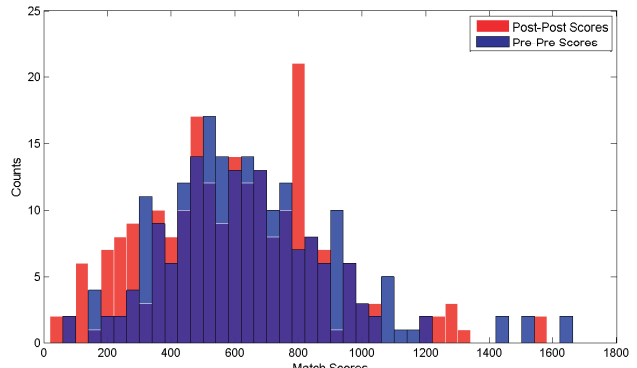

(a)

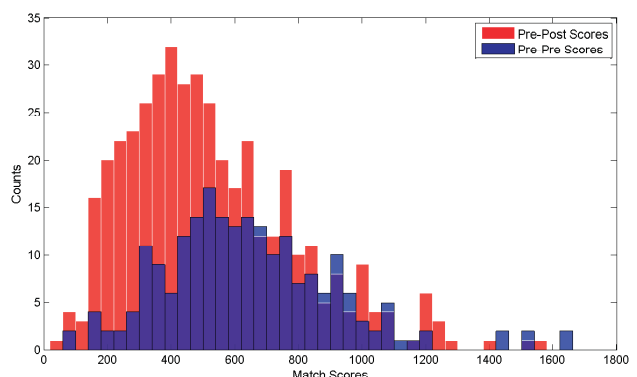

(b)

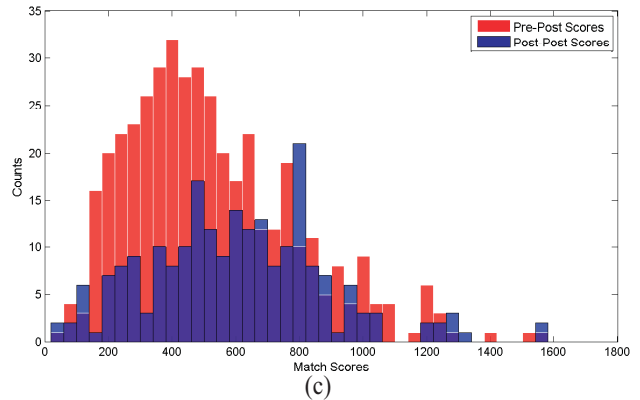

Figure 4. Genuine match score distribution obtained using VeriEye. (a) Gallery and probe images are either pre or post alcohol consumption images, (b) and (c) comparison with match score distribution when the gallery is pre alcohol image and the probe is post alcohol consumption image. The pre-post distribution is shown with pre-pre and post-post distribution to clearly represent the change in distribution.

relatively smaller scale of measurements.

- In case of dilation, the average extent of change, $E(S)$, is observed to be 1.18 with a standard deviation of 0.17 , whereas in the case of contraction, the average is 0.86 with a standard deviation of 0.13 . Figure 5 illustrates the values of "extent of change" for the pre and post iris comparisons using iris segmentation algorithm described in [20]. The figure shows that there are several extreme cases where pupil area varies by a large extent. Figures 6 and 7 show two examples of extreme cases where both the algorithm in [20] and VeriEye matchers fail to match the iris images captured pre and post alcohol consumption. 


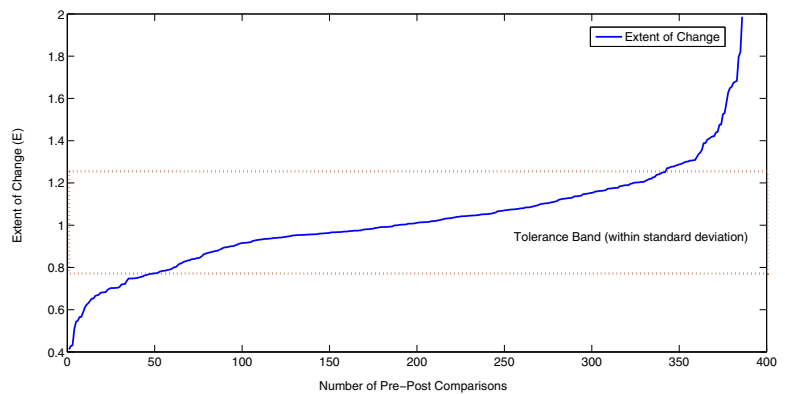

Figure 5. Illustrating the extent of pupil change, $E$, calculated using Equation 6. The band (between the two dashed red lines) represents the tolerance limit of iris recognition algorithms, i.e., if the value of $E$ lies outside the tolerance band, then the confidence of the match is significantly reduced.
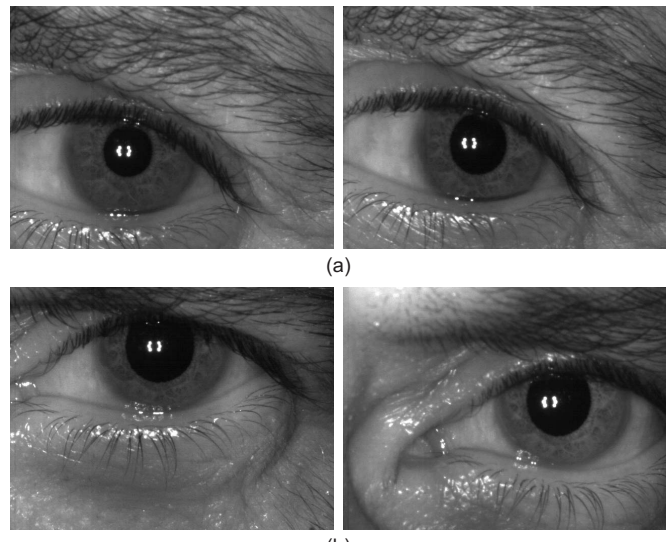

(b)

Figure 6. Example of dilation case: (a) pre alcohol images and (b) post alcohol consumption images of two different irides. VeriEye match scores for pre alcohol images is 652 and post match score images is 396. On matching pre alcohol image with post alcohol image, VeriEye gave a score of 184. Similarly, 2D log polar Gabor based algorithm generates pre alcohol match score of 0.22 , post alcohol match score of 0.14 and pre-post alcohol match score of 0.35 . Note that VeriEye generates similarity scores whereas Vatsa et al.'s algorithm [20] generates distance scores.

- The extent of change is observed to deviate from the standard deviation tolerance band in around $28 \%$ of the cases and the confidence of both the recognition algorithms goes down significantly for such cases. In other words, on the IIITD-IUAI database match scores of one in every five subjects differs significantly due to alcohol consumption.

- As shown in Figures 3(a) and (b), the overlap between genuine and impostor match score distributions is very small. On the other hand, when the gallery consists of pre alcohol consumption images and the probe consists of post alcohol consumption images, the overlap

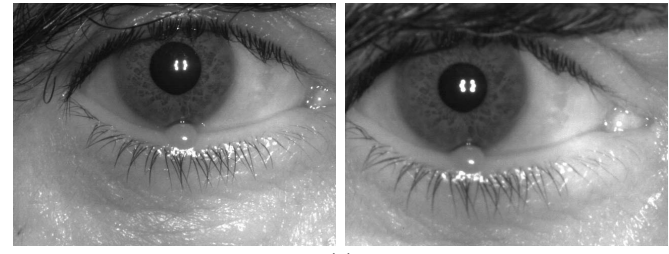

(a)

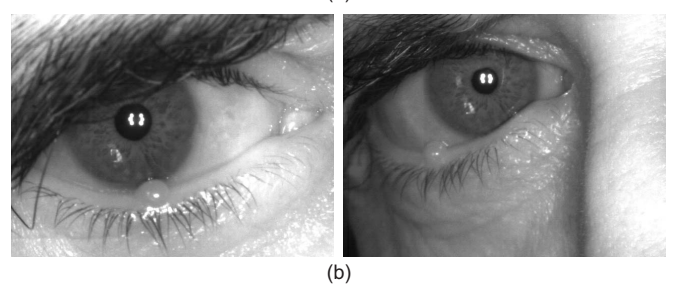

Figure 7. Example of constriction case: (a) pre alcohol images and (b) post alcohol consumption images for two different irides. VeriEye match scores for pre alcohol images is 1135 and post alcohol image match score is 371 . On matching pre alcohol image with post alcohol image, VeriEye failed to generate the match score. Similarly, 2D log polar Gabor based algorithm generates pre alcohol match score of 0.27 , post alcohol match score of 0.19 and pre-post alcohol match score of 0.33 .

increases by about $20 \%$. These cases correlate with the cases observed in Figure 5 and demonstrate that alcohol influence has significant effect on the performance of iris recognition.

- As shown in Figure 4, the match scores obtained from VeriEye approximately follow a Gaussian distribution with a mean of 500 when both the gallery and probe images are either before or after alcohol consumption. However, the histogram becomes right skewed and the mean shifts to 400 for the match scores obtained when the gallery is captured before consumption and the probe image is captured after consumption. This significant right-skew with a mean shift of 100 shows a drastic reduction in the confidence of match scores of the VeriEye matcher for such cases. For genuine scores, at $95 \%$ confidence level, VeriEye yields the correct verification accuracy of $99.1 \%$ (1.8\% margin of error) with pre alcohol consumption images, $98.6 \%$ ( $2.2 \%$ margin of error) with post alcohol consumption images, and $97.7 \%$ (2.8\% margin of error) with prepost alcohol consumption iris images ${ }^{6}$. Though the verification accuracy is not much affected, the match scores obtained from VeriEye show less confidence in matching pre-post alcohol consumption images.

- The Receiver Operating Characteristics (ROC) curves in Figure 8 show that if both the gallery and probe images are captured without alcohol, the algorithm yields

\footnotetext{
${ }^{6}$ Note that VeriEye's SDK does not provide the license to generate match scores for impostors.
} 


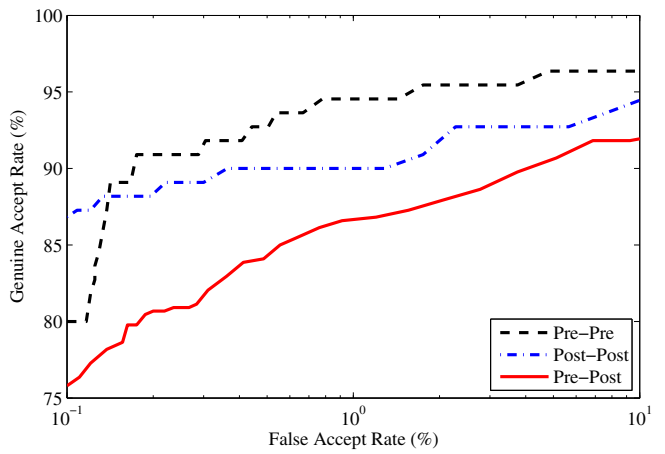

Figure 8. ROC curve (on log scale) to illustrate the performance of iris matching algorithm in [20] for the three cases when gallery and probe images are captured (i) before alcohol consumption, (ii) after alcohol consumption, and (iii) gallery is before while probe is after consumption.

the best performance. The reduction in performance is observed when the gallery and/or probe are captured after alcohol consumption. The ROC curve for matching pre and post alcohol consumption is in accordance with the match score distribution and shows significant reduction in verification accuracy of the iris recognition algorithm by Vatsa et al. [20].

\section{Conclusions}

There are several substances such as alcohol, LSD, MDMA, cocaine, and marijuana that affect iris property. Physiologically, these substances temporarily dilate or constrict pupil to a large extent. Iris recognition of a person under influence of these substances, therefore, can be viewed as a form of attack on the integrity of a biometric system. This paper shows that alcohol influence is a new covariate in iris recognition that affects the matching performance significantly. The experiments performed on the IIITD Iris Under Alcohol Influence database suggest that after alcohol consumption, usable iris area changes due to deformation caused by dilation or constriction. This change is dynamic and varies from person to person. This is similar to disguise covariate in face recognition where the appearance can be changed provisionally. This covariate can be viewed as a vulnerability of iris recognition systems. It is our assertion that next generation iris recognition systems should consider this covariate and enhance their capabilities to address such challenges.

\section{Acknowledgement}

The authors would like to thank the subjects who participated in this study.

\section{References}

[1] http://www.nicd.us/ signsandsymptomsofuse.html.

[2] http://www.irisguard.com/pages.php?menu_ id $=33$.

[3] http://www.pcworld.com/article/141697/.

[4] NEXUS. http://www.cbp.gov/xp/cgov/ travel/trusted_traveler/nexus_prog/ nexus. xml.

[5] UIDAI. http://uidai.gov.in/.

[6] UK Iris. http: //www.ukba.homeoffice.gov.uk/ customs - travel/Enteringtheuk/usingiris/.

[7] VeriEye. http://www.neurotechnology.com/ verieye. html.

[8] W. Boles and B. Boashash. A human identification technique using images of the iris and wavelet transform. IEEE Trans. on Signal Processing, 46(4):1185-1188, 1998.

[9] K. W. Bowyer, K. Hollingsworth, and P. J. Flynn. Image understanding for iris biometrics: A survey. Computer Vision and Image Understanding, 110(2):281-307, 2008.

[10] B. Brown, A. Adams, G. Haegerstrom-Portnoy, R. Jones, and M. Flom. Pupil size after use of marijuana and alcohol. American Journal of Ophthalmology, 83(3):350-354, 1977.

[11] J. Daugman. High confidence visual recognition of persons by a test of statistical independence. IEEE Trans. on Pattern Analysis and Machine Intelligence, 15(11):1148-1161, 1993.

[12] J. Daugman. New methods in iris recognition. IEEE Trans. on Systems, Man and Cybernetics - B, 37(5):1168-1176, 2007.

[13] L. Flom and A. Safir. Iris recognition system, 1987. United States Patent 4,641,349.

[14] C. A. Franklin. Medical Jurisprudence and Toxicology. 21st edition, 1988.

[15] N. Kalka, J. Zuo, N. Schmid, and B. Cukic. Estimating and fusing quality factors for iris biometric images. IEEE Trans. on Systems, Man and Cybernetics - A, 40(3):509-524, 2010.

[16] L. Ma, T. Tan, Y. Wang, and D. Zhang. Personal identification based on iris texture analysis. IEEE Trans. on Pattern Analysis and Machine Intelligence, 25(12):1519-1533, 2003.

[17] D. Monro, S. Rakshit, and D. Zhang. Dct-based iris recognition. IEEE Trans. on Pattern Analysis and Machine Intelligence, 29(4):586-596, 2007.

[18] J. E. Richman, K. G. McAndrew, D. Decker, and S. C. Mullaney. An evaluation of pupil size standards used by police officers for detecting drug impairment. Clinical Research, 75(3), 2004.

[19] A. Ross. Iris recognition: The path forward. IEEE Computer, pages $30-35,2010$.

[20] M. Vatsa, R. Singh, and A. Noore. Improving iris recognition performance using segmentation, quality enhancement, match score fusion, and indexing. IEEE Trans. on Systems, Man, and Cybernetics - B, 38(4):1021-1035, 2008.

[21] R. Wildes. Iris recognition: an emerging biometric technology. Proceedings of the IEEE, 85(9):1348-1363, 1997. 